\title{
Methodology to Define Delivery Accuracy Under Current Day ATC Operations
}

\author{
Shivanjli Sharma ${ }^{1}$ and John E. Robinson, III $^{2}$ \\ NASA Ames Research Center, Moffett Field, CA, 94035
}

In order to enable arrival management concepts and solutions in a NextGen environment, groundbased sequencing and scheduling functions have been developed to support metering operations in the National Airspace System. These sequencing and scheduling algorithms and tools are designed to aid air traffic controllers to develop an overall arrival strategy. The ground systems being developed will support the management of aircraft to their Scheduled Times of Arrival (STAs) at flow-constrained meter points. This paper presents a methodology for determining the undelayed delivery accuracy for current day air traffic control operations. The new method supports the definition of metrics that will allow near-future ground automation tools to successfully achieve desired separation at meter points, enabling aircraft to meet their STAs while performing Performance-Based Navigation (PBN) procedures in the terminal area. The research develops metrics for estimating the undelayed delivery accuracy of arrival flights as they cross the arrival meter fix. The metric is based on measurement of delivery performance and analysis of Estimated Time of Arrival (ETA) accuracy for the Federal Aviation Administration's (FAA) Time-Based Flow Management (TBFM) system. This method utilizes a wide set of data from the Operational TMA/TBFM Repository (OTTR) system, which processes raw data collected by the FAA from operational TBFM systems. A large data set was used which consisted of all the traffic across a two month period in 2013, the hottest and coldest months, arriving into four airports: George Bush Intercontinental Airport, Denver International Airport, Los Angeles International Airport, and Phoenix Sky Harbor Airport. The new method of automated analysis provides a repeatable evaluation of undelayed delay metrics for current day traffic, new releases of TBFM, enhancements to TBFM, and across different airspace environments.

\section{Introduction}

$\mathrm{T}$ he Federal Aviation Administration (FAA) and NASA have been collaborating on the development of a nearterm ground-automation system in order to support metering operations throughout the National Airspace System (NAS). This near-term solution extends and enhances the FAA's Time-Based Flow Management (TBFM) system. TBFM is a time-based scheduling tool, which is currently in use at En Route Air Route Traffic Control Centers (ARTCC) facilities throughout the NAS. TBFM assists air traffic controllers and traffic managers in matching arrival demand with airport arrival constraints such as required separations and Airport Arrival Rate (AAR). It achieves this by providing recommended sequencing, scheduling and spacing information for arriving aircraft to the TRACON Gate through the generation of scheduled times of arrival (STA) at the meter fix, merge fix and runway [1].

These terminal sequencing and scheduling (TSS) tools and their enhancements become increasingly important as future Next Generation Air Transportation System (or NextGen) technologies and operations are being implemented. NextGen includes goals for expanding the capacity of high-demand airports, while increasing the fuel efficiency of arriving aircraft. Today, arrivals into high-density airports during high throughput time periods often experience significant inefficiencies resulting from the use of static miles-in-trail procedures, step-down descents, and significant vectoring close to the airport [1]. TSS, the suite of tools developed by NASA, creates a single, integrated arrival solution. The tools include an enhanced version of TBFM which extends the basic TBFM scheduling capability by including merge fixes inside the TRACON airspace. This prototype of TBFM also includes

\footnotetext{
${ }^{1}$ Research Aerospace Engineer, Aviation Systems Division, NASA Ames, Mail Stop 210-1, AIAA Member

${ }^{2}$ Chief Engineer, Aviation Systems Division, NASA Ames, Mail Stop 210-1, AIAA Senior Member.
} 
an improved terminal delay model that more accurately represents Performance-Based Navigation (PBN) based trajectories. This suite of tools also includes a set of Controller-Managed Spacing (CMS) decision support tools for TRACON controllers to manage aircraft delay better using speed control. The third component is flight-deck interval management (FIM), which consists of aircraft avionics and flight crew procedures to conduct arrival-toarrival airborne spacing operations. TSS includes sequencing and scheduling functions and tools to help air traffic controllers in managing aircraft to meet their scheduled time of arrival (STA) at meter points. The sequencing and scheduling algorithms and tools are designed to aid air traffic controllers in developing an overall arrival strategy.

To enable enhanced terminal area operations and ensure that terminal sequencing and spacing can occur effectively, metering delivery accuracy requirements are integral and have been targeted as an area of improvement. The delivery of flights to meter fixes required further investigation to determine what was needed to achieve the level of delivery accuracy needed to support the efficiency benefits of proposed NextGen operations. Research in this field is limited and thus far there has been little effort to determine how delivery accuracy metrics can be defined and calculated given the current data available, and to quantify delivery accuracy values based on current day ATC operations, regardless of whether TBFM was being utilized. Both of these were necessary to understand how delivery accuracy requirements could be further refined to ensure that when TSS is implemented the maximum benefits of the tools can be realized.

The performance of arrival management systems has been analyzed in various contexts. In reference [6], the authors analyzed trajectory uncertainties to determine the minimum targeted spacing at the terminal meter fix on the en-route/terminal boundary. This work considered aircraft continuing their Area Navigation (RNAV) arrivals to the runway without controller intervention. In the work, the aircraft were metered to the terminal meter fix by conventional means, and errors in the inter-arrival time were modeled. That methodology was used to establish inter-arrival spacing for flight tests conducted at Louisville International Airport (SDF), and in reference [8], the performance predicted by the model was shown to agree well with measured performance. The delivery accuracy of STAs for saturated metering operations at Hartsfield-Jackson Atlanta International (ATL) operations was analyzed in reference [8]. With a similar motivation to the research in reference [6], the authors determined that a tighter, close to zero, delivery accuracy at the meter fix was required to achieve certain efficient operations (i.e., no vectoring or extended final) in the terminal area. A tool was developed and applied in reference [9], which combined the effects of metering to the terminal meter fix and the runway, scheduled delay, and controller intervention rates to optimize the performance of a scheduler for the terminal area.

Current methods of delivery accuracy evaluation, as referenced above, focused on small sample sizes as the ETA accuracy was computed for only undelayed flights, with limited ATC intervention. The objective of this research was to formulate a methodology to perform TBFM undelayed delivery accuracy analysis using existing data for current day operations across a large set of aircraft. The research developed metrics for estimating the undelayed delivery accuracy for arrival flights based only on flight times for aircraft that have arrived at the meter fix uninterrupted by air traffic control. Identification of uninterrupted flights was estimated by comparing scheduled times of arrival to estimated times of arrival and making the assumption that a difference of less than 30 seconds could be reasonably assumed to mean uninterrupted. This method allowed a broader understanding of time-ofarrival errors rather than focusing on a small set that relied on the physical modeling of the aircraft, conformance to arrival routes, and ATC intervention. In addition, this research defines a possible ETA accuracy requirement for TSS while also illustrating a set of potential results for NAS performance improvements and understanding the impact of new tools that that may be implemented in the future.

This paper is organized as follows: Section II describes background of delivery accuracy overall; Section III describes the design of the new method to analyze the undelayed delivery accuracy at meter points; Section IV examines the relation between the calculated metric findings and results across four airport sites; Section V concludes the paper with a summary of key findings.

\section{Approach}

One of the basic capabilities of TBFM is the computation of the estimated time of arrival (ETA) to the meter fix and runway for each arrival aircraft in the airspace. Based on these ETAs, TBFM computes the sequences and scheduled times of arrival (STAs) to the meter fix and runway for each aircraft to meet the sequencing and scheduling constraints entered by the user $[12,13]$. The ETAs are calculated based on the four-dimensional (4D) trajectories predicted for each aircraft. The key functions in the ground automation are trajectory modeling, sequencing and scheduling, and schedule conflict prediction and resolution. Prior to a freeze horizon, the ETAs are used as input to the sequencing and scheduling function to determine a de-conflicted schedule at the meter fix. Typical and current TBFM performance measurements focus on the accuracy of the predicted ETAs for a sample of 
flights not impacted (interrupted) by any ATC intervention. An uninterrupted flight can be defined as a flight that has not received any clearance from ATC, lateral, vertical or speed-related, that will change its objectives after it has passed the freeze horizon. It is difficult to identify flights that were not impacted by ATC intervention in recorded operational data as these clearances are in general not recorded or easily analyzable. However, for this work a broader approach was constructed that did not base filtering solely on determining whether a flight was interrupted. Instead, flights were analyzed over a broad period of time using various filters to gauge, regardless of direct ATC intervention, nearly-undelayed delivery accuracy to the meter fix.

The existing data sets and tools were surveyed in order to determine if they could be leveraged to compute the desired metric across a large set of airports and days. One tool that was evaluated was the TMA ETA Evaluation Tool developed by Engility with NASA Ames. The tool provided analysis abilities including the capability to produce cone test plots that illustrate ETA accuracy along with the STA accuracy, which is calculated as the difference between the last frozen STA and the actual meter fix crossing time for all the flights in the sample. The plots produced by this tool are intended to give a measure of how accurate the frozen STAs presented to the controllers are versus the actual crossing of the meter fix. Despite the functions this tool provides, it proved time intensive to use across a wide set of data that was needed to get an accurate picture of operations across several airports. The tool was designed to exclude any aircraft that were given controller interventions, which significantly limited the final data set that was produced for analysis as any aircraft that deviated from its route was excluded. Based on this experience, it was determined that utilization of another resource of aircraft landing and scheduling reports would be more conducive to obtain data needed for this particular analysis. However, this would require the creation of a new processing and filtering algorithm.

\section{A. Data Set Used for Analysis}

As a result of a survey of existing tools and data sets, it was determined that using a wide set of data from the Operational TMA/TBFM Repository (OTTR) system would be a more favorable way to obtain the data needed for this particular analysis. The NASA NTX Data Collection System (NTXDCS) runs a daily process, which collects all operational TBFM Communications Manager (CM), and Input Source manager (ISM) data from William J. Hughes Technical Center (WJHTC). The WJHTC data is binary formatted messages of all data passed between TBFM components [7]. The NTXDCS system obtains, processes and makes TBFM data available. The OTTR system generates daily reports concerning ATC status, intent and actions. Due to its availability, ease of use, and vast collection of data across several airspaces, it was determined that the OTTR data set would be the most useful to move forward with in this analysis. The particular variables needed for further analysis were determined along with the necessary OTTR reports, by working closely with the repository team, additional analysis reports were developed that provided key ETA and STA information at the freeze horizon. One major benefit of the OTTR data is that the data across several airports could be analyzed over large periods of time.

The OTTR data processes and stores TBFM data daily in various formats across several airspaces. This allowed the development of parsing methods and raw data processing that would not rely on other computationally expensive tools. The majority of this work consisted of the development of the ability to filter flights to create a subset of aircraft that could be considered undelayed, which is defined as a flight at the freeze horizon with an ETA and STA difference that was minimal or close to zero. This was a broad method that allowed the consideration of a large data set which consisted of all the traffic across a two month period in 2013 (the hottest and coldest months) arriving into four airports. The four airports considered include George Bush Intercontinental Airport, Denver International Airport, Los Angeles International Airport, and Phoenix Sky Harbor Airport. The results from this data set illustrated the undelayed STA delivery error versus the actual meter fix crossing time, and the distribution of these delay values across each of the airports for both the hottest and coldest months.

\section{B. Data Considerations}

The FAA TBFM system has several methods that allow metering to be turned on and off. Metering can be turned on/off at the airport level or ARTCC level. Along with the expansion of Adjacent Center Metering (ACM), this requires each ARTCC to enable/disable metering at each remote metering location. An additional complication to determining when metering is occurring is the status of the local and host computers and more importantly, accounting for whether metering lists are actually displayed on controller scopes. The OTTR repository does contain a metering usage report that collects times when metering was turned on and off at the ARTCC level but no method to determine if metering lists were displayed to the controller currently exist. Rather than attempting to account for all these required pieces of information in determining whether or not metering is occurring, a broader approach at viewing the data was constructed. 
A significant part of the work presented in this paper relies on automating the filtering approach to capture flights that have passed the meter fix uncontrolled by ATC from recorded data. Flights are considered uncontrolled if the difference between the scheduled time of arrival and estimated time of arrival is within 30 seconds. Currently, no method for identification of flights using this definition exists to perform this filtering method. The use of this data is presented in the next section.

\section{Method to Compute Delivery Accuracy}

The method analyzes the undelayed delivery accuracy at meter points in order to understand changes of desired flow rates as well as enabling the definition of metrics. This metric is referred to as "nearly undelayed delivery accuracy." This method was created to be used in an automated fashion and provides a repeatable evaluation of the delay metrics for current day traffic. By creating a simple filtering methodology, this also allowed for the definition of a delivery accuracy metric for use in understanding current day and future arrival operations without necessarily being constrained to analyzing periods of time when TBFM and metering lists are visible to controllers on their ATC displays.

This method viewed airport data collected from the OTTR system across a broad period of time (a month of data) and did not take into account if metering, direct ATC intervention, or other changes to aircraft trajectory were occurring. Instead, by looking at the broad set of data and constraining only based on a few parameters, an initial view of delivery accuracy for nearly undelayed aircraft was identified. This section details the OTTR reports that were utilized for this analysis followed by the filtering methodology that was developed.

\section{A. OTTR Reports Analyzed}

The following reports from the OTTR warehouse were utilized: the airport delay report, the flow setting and metering usage report, and the landed and frozen report. The next sections contain a brief description of the reports and details of the data.

\section{Airport Delay Report}

The airport delay report allowed the method to leverage accurate ETA and landing time data in order to calculate the amount of delay incurred within the ARTCC. Other measurements of ETAs typically utilize straight-line paths into the airport rather than the aircraft's actual route; however, as these data are based on TBFM calculations, its ETAs account for routing information, detailed aircraft-specific trajectory modeling information and current airport configuration data in generating its ETAs [7]. Ultimately, utilizing this report allowed the ETAs to be based on more realistic flight times and delay values.

The delay report is based on the TBFM Delay Reporting System (DRS) logic [7], which essentially defines delay as the actual landing time and original ETA difference. The original ETA is the ETA of an aircraft as it enters an ARTCC. To prevent errors in the ETA estimate, the OTTR system takes the average of the first five ETAs after an aircraft has crossed the ARTCC boundary and utilizes this ETA as the original ETA value. An aircraft that lands later than this original ETA value is given the associated delay value in the report. The OTTR report includes a landing time that makes use of a landing zone algorithm; this logic is adapted for each airport with a particular radius and altitude that an aircraft must be present in for a certain duration of time in order to be given a landing time.

This report also maintains a requirement for the flight to be within the destination Center boundary and is also part of the definition of delay per the delay reporting system. This definition for later research may be reworked in order to account for the delay which is incurred outside of the Center boundary when given adjacent center metering. Nevertheless, from an operational reporting standpoint this definition is used as it is only required to report when flights have experienced delay in a particular center's airspace.

The airport delay report also calculates the amount of delay within the Center by taking the average of the first five ETAs to each meter fix (MF) reference point, such as MF or runway, and stores this value. These values can then be compared with actual crossing times to these reference points.

Table 1 below provides a sample of the Airport Delay Report. The header field is included for reference; note that not all fields are replicated here due to space. 
Table 1. Sample of OTTR Airport Delay Report

\begin{tabular}{|c|c|c|c|c|c|c|c|c|c|c|c|c|}
\hline 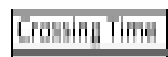 & Arpanth & 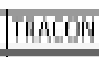 & Aramp & 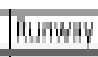 & | HRT TWE & 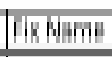 & Drighth & 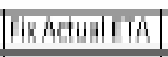 & minting & 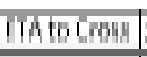 & 46 대애 & 4T TVE \\
\hline 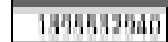 & MWF". & 阴 & 阴算 & 911 & MF Gif & Mดแ & nhen & hinti & 㱚 & Whiti. & ตัด⿴囗十⺝ & BAM \\
\hline 1971 & 14.9 & |min & Pна & 21 & MF & 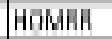 & if & ดั & 111 & hath & hiti & $1 \mathrm{AH}$ \\
\hline 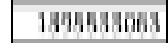 & 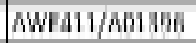 & "मй & 阴脱 & 211 & MF 5 酣 & PMFT & and & hнй & 14 & แม⿵冂卄 & MัM⿰ & BH \\
\hline 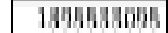 & 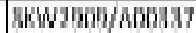 & | & PHW & III & MF $5 \mathrm{EF}^{\mathrm{N}}$ & HคUKF & has & ing & m & whing & the & |ithI \\
\hline
\end{tabular}

\section{Flow Settings Report and Metering Usage Report}

The flow settings report contains information about the freeze horizons for each airport and the metering usage report provides a flag indicating whether metering was turned on for that particular center. However, due to the caveats mentioned in this paper regarding the metering usage at individual ARTCCs and TRACONs, this report is described here for future reference. For a more complete filtering methodology, this report may be leveraged to obtain delivery accuracy data if the issues around the metering flag and its implications can be resolved.

Table 2 below provides a sample of the Flow Settings Report, the header field is included for reference; note that not all fields are replicated here. A flow acceptance rate of 3600 indicates an unrestricted rate.

Table 2. Sample of OTTR Flow Settings Report

\begin{tabular}{|l|l|l|c|c|r|r|}
\hline Time & Date & Tracon Name & Manually Scheduled & Reschedule All Aircraft & Start Time Offset & TRACON Flow Acceptance Rate \\
\hline 1393668001 & $3 / 1 / 14$ & P50 & FALSE & FALSE & 0 & 3600 \\
\hline
\end{tabular}

\section{Landed and Frozen Report}

The landed and frozen report is an additional analysis report that was added to the OTTR repository. This report captures the delay for flights and includes information about the ETA to the meter fix, the STA to the meter fix, as well as aircraft state information such as distance from the meter fix and speed when considered crossing the meter fix. In addition to typical arriving flights, this report also includes internal departures. All flights are populated at least once, as soon as a landing time is given based on the landing zone logic. Some flights do appear twice; the flights that appear twice occur when freeze horizons are turned on, typically during metering. Some flights are frozen immediately upon turning on the freeze horizons, similar to TBFM being brought on while flights are already within the freeze horizon.

Table 3 below provides a sample of the Landed and Frozen Report, the header field is included for future reference, note that not all fields are replicated here. The columns are broken into two sections to illustrate several of the fields.

Table 3. Sample of Landed and Frozen Report

\begin{tabular}{|c|c|c|c|c|c|c|c|c|c|c|c|}
\hline mat macond & Ariant & Alitio & Transh & BL TYË & | Des Alpout & Arr hrosent & | rarant & Antivit & 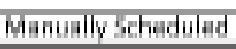 & P地 & 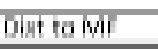 \\
\hline 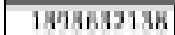 & PHH & Hown & mh & Ehi & BIYLL & HPHK & Fas & YFA & 슬 & Pan & 1.776เมตร \\
\hline 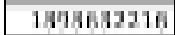 & PHH & HWh & Phin & 1 & BMEW & HPHX & Wh & YEN & 내믄 & WH & แดหมกา \\
\hline
\end{tabular}

\begin{tabular}{|l|c|r|l|l|r|l|}
\hline Runway Name & Runway ETA & Runway STA & Scheduling MF & MF ETA & MF Sched STA & Crossed MF \\
\hline \hline $25 \mathrm{~L}$ & 1393632148 & 1393632152 & PAYNT & $1.394 E+09$ & 1393631203 & YES \\
\hline 26 & 1393632219 & 1393632224 & HOMRR & $1.394 \mathrm{E}+09$ & 1393631525 & YFS \\
\hline
\end{tabular}

\section{B. Filtering and Processing Methodology}

To meet the objective of creating an automated, repeatable approach to analyze nearly undelayed delivery accuracy that is applicable to any airspace in the NAS, a series of MATLAB scripts were developed. These scripts parsed the necessary OTTR reports, as given above, and selected applicable aircraft. The first step involved creating MATLAB scripts that automated the loading of all the three OTTR reports listed above across the desired time frame, which in this case was one-month periods.

The second and most substantial task was to automate the filtering of a set of flights that focused on aircraft with a difference between ETA (estimated time of arrival to the meter fix) and STA (scheduled time of arrival to the meter fix) of less than \pm 30 seconds, as shown in Equation (1) below.

$$
-30 \mathrm{sec}<E T A-S T A<+30 \mathrm{sec}
$$


Eq. (1) approximates the definition of nearly undelayed aircraft, as this analysis limits the aircraft to those that were most likely undelayed. The 30 second threshold in Eq. (1) represents a reasonable assumption of undelayed aircraft because when and if delay values are shown on controller displays in most ARTCCs the delay values appear as truncated minute values not rounded or in tens of seconds. Therefore, if an aircraft has a delay of $+/-59$ seconds, it will be displayed to an air traffic controller as a delay of zero. Hence, all aircraft that have delay values of less than 30 seconds, can be considered "nearly undelayed."

The automated filtering algorithm then cross references the Landed and Frozen Report. The goal was to filter and remove aircraft from the set that did not actually cross the meter fix or land at the airport. There were several critical messages for the filtering methodology, some of which are outlined here. The first field utilized was Fix Type. All aircraft that were given any other fix type scheduling (outer arc, runway) except those aircraft scheduled to the meter fix display point will filtered out. The display meter fix and scheduling fix are the same in most cases. Since there are circumstances where they differ, this may be important in down stream analysis. For instance, sites that use some other complex operational arrangement may utilize various fix types for scheduling; therefore, the display meter point in the report data was used. In addition to the above filtering, any aircraft that were manually scheduled or contained inactive values were removed.

The full aircraft identification numbers were used to pull crossing times at the meter fix from the Landed and Frozen report. These crossing times were converted from a standard date and time to a UTC value that could be used for a delay computation. Once a full set of flights that met the above criteria were obtained, a final down selection of the flights was conducted using the aircraft type field; all aircraft that were not jets were removed, so all turboprops were filtered from the set of aircraft. Finally, using this set of aircraft, the delivery accuracy was computed for all aircraft across the month time period considered. Equation (2), below, defines the delivery accuracy calculation.

$$
\text { STA - ATA }
$$

Eq. (2) shows that by taking the difference of the STA at the meter fix and the ATA, where the ATA represents actual time of arrival to the meter fix, the undelayed delivery accuracy of a down-selected set of aircraft can be shown across airports in the NAS and across periods of time. This method was utilized across four airports and across the warmest and coldest months of 2013, the results of which follow in the next section.

\section{Airport and Weather Selection}

The four airports selected to test this filtering methodology include George Bush Intercontinental Airport (IAH), Denver International Airport (DEN), Los Angeles International Airport (LAX), and Phoenix Sky Harbor Airport (PHX). These airports were chosen as they represented potential key sites for future testing of new ground-based TSS tools. This set of tools has undergone significant development at NASA [1,2,3,4] over the last several years and has recently been included in the FAA's future investment in TBFM. Each of these airports have local ARTCC metering in place, utilize optimal profile descents (OPDs), and have an airspace structure that would lend itself to initial testing of TSS.

The automated filter was applied to a two month data set across each of the four airports in 2013. This was a broad method that considered a large data set which consisted of all the traffic across the hottest and coldest months for each of the airports. The hottest/coldest months were chosen because the en route winds are typically highest in the coldest months and lowest in the warmest months. The hottest and coldest months were chosen through a survey of the weather information at each airport across the year of 2013. The hottest and coldest values indicate either the hottest or coldest average temperature across a month. The method thus provided a snapshot of different weather and wind conditions that might impact the undelayed delivery accuracy into each of the airports.

\section{Initial Results}

Section III provided information regarding the OTTR data reports that were used for this analysis and also detailed the steps in the filtering method. This section presents the numerical data across the airports and is given in Table 4, which provides the data across each of the subsequent filtering steps for each of the airports and across the two months given. The initial number of aircraft is given in the table in the first row, but the first major step of down-selection to create a set of nearly undelayed aircraft can be seen in the second row. The third row illustrates 
the matching of full aircraft identifiers across the Airport Delay as well as the Landed and Frozen Report. The fourth row displays the final step of automated filtering where the fields discussed above are removed along with any turbojets. Finally, below the table the percentage of remaining aircraft from the full set of each month is displayed.

Table 4. Filtering Methodology and Resulting Aircraft across Four Airports.

\begin{tabular}{|c|c|c|c|c|c|c|c|c|}
\hline & \multicolumn{2}{|c|}{ DEN } & \multicolumn{2}{|c|}{$\mathrm{IAH}$} & \multicolumn{2}{|c|}{ LAX } & \multicolumn{2}{|c|}{ PHX } \\
\hline & August & December & August & December & September & January & June & January \\
\hline Initial Set of Aircraft & 7406 & 2561 & 1263 & 7369 & 34503 & 28613 & 10968 & 13597 \\
\hline $\begin{array}{l}\text { Filtered by an error of } \\
(\text { ETA-STA }< \pm 30)\end{array}$ & 4177 & 1419 & 804 & 4944 & 8913 & 7576 & 6892 & 8703 \\
\hline $\begin{array}{l}\text { Filtered by matching aircraft } \\
\text { id to Landed and Frozen } \\
\text { Report }\end{array}$ & 2517 & 1167 & 398 & 1796 & 2457 & 2867 & 1504 & 3799 \\
\hline $\begin{array}{l}\text { Filtered turbos, non-active, } \\
\text { and manually scheduled }\end{array}$ & 2336 & 933 & 366 & 1688 & 2248 & 2637 & 1351 & 3404 \\
\hline $\begin{array}{l}\text { Percent of Aircraft } \\
\text { Remaining From those } \\
\text { frozen }\end{array}$ & $31.54 \%$ & $36.43^{\circ} /$ & $28.98 \%$ & $22.91 \%$ & $6.52 \%$ & $9.22 \%$ & $12.32 \%$ & $25.03 \%$ \\
\hline
\end{tabular}

Table 4 illustrates that while still considering a minor subset of undelayed aircraft the number of aircraft that can be analyzed still represents a substantial percentage of total aircraft, up to $36.43 \%$. Of the airports analyzed DEN and IAH produced a larger percentage of aircraft remaining after the filtering process. This requires further investigation into the airport configuration and landing at nearby airports. Given the LAX filtering values, it can be seen that the largest drop in the number of aircraft occurred between the initial undelayed filtering and the matching across the OTTR reports. This could indicate landing at nearby airports may be reducing the number of aircraft that can be matched to those actually landing at LAX as specified by the Landed and Frozen report.

The results from this data set were used to compute the undelayed STA delivery error versus the actual meter fix crossing time, and the distribution of these delay values across each of the airports. This analysis also illustrated a definition of an undelayed delivery accuracy metric for use in understanding of current day and future arrival operations. The results of the automated filtering support the decision to calibrate the values to include a \pm 30 second threshold to create a subset of nearly undelayed aircraft. The majority of flights are filtered because of landing at nearby airports and the rest are reduced due to the third set of filtering which removes several of the smaller criteria. Further investigation into the filtering by cross referencing the OTTR reports is required to definitively assess the method. The second most number of flights are removed primarily due to non active aircraft and the presence of turbojets.

For each airport across each month, a histogram showing the nearly undelayed delivery accuracy distribution was produced along with a cumulative distribution function (CDF) of the data. A subset of these data can be seen in Fig. 1 which displays the histograms for all four airports during the hottest months of 2013. In addition to the histograms, statistical data were extracted Table 5. Undelayed Delivery Accuracy Mean and Standard Deviation

\begin{tabular}{|c|c|c|c|}
\hline intwin & Mhinilli & Meing | & 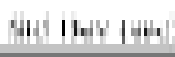 \\
\hline \multirow[t]{2}{*}{ INH } & Man & in ini & 4 \\
\hline & I His & i.lii & I i \\
\hline \multirow[t]{2}{*}{ IAII } & Aнill & In & 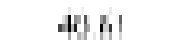 \\
\hline & then & H & alit \\
\hline \multirow[t]{2}{*}{ I A h } & Fin & 17 int & H.11 \\
\hline & ilin & $=17 \mathrm{~T}$ & 9714 \\
\hline \multirow[t]{2}{*}{ IPIM } & Iining & i & A 4 H \\
\hline & IHin & 297.74 & II hil \\
\hline
\end{tabular}

from each of the sets collected. The undelayed delivery accuracy mean and standard deviation for each airport, across each month, can be seen in Table 5. As seen in Fig. 1 and Table 5, it can be noted that during August of 2013, Denver International Airport, had a computed average undelayed delivery accuracy of 11.03 


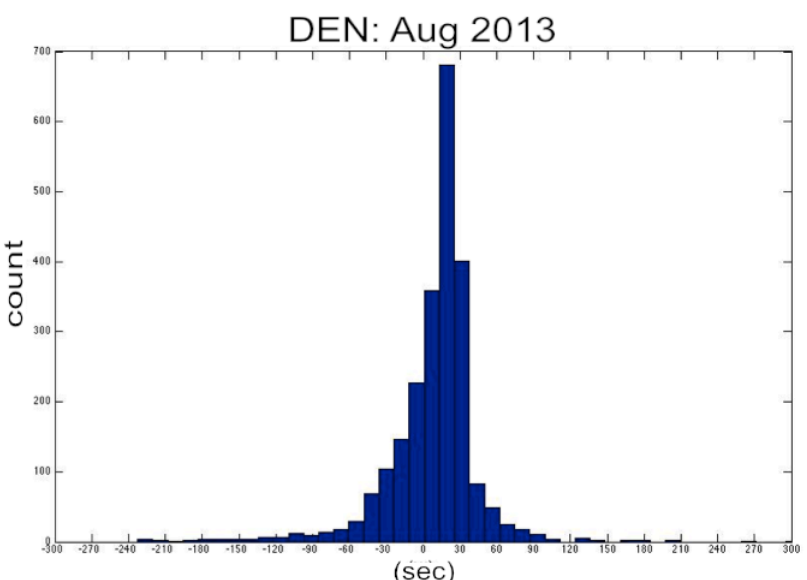

$(\mathrm{sec})$

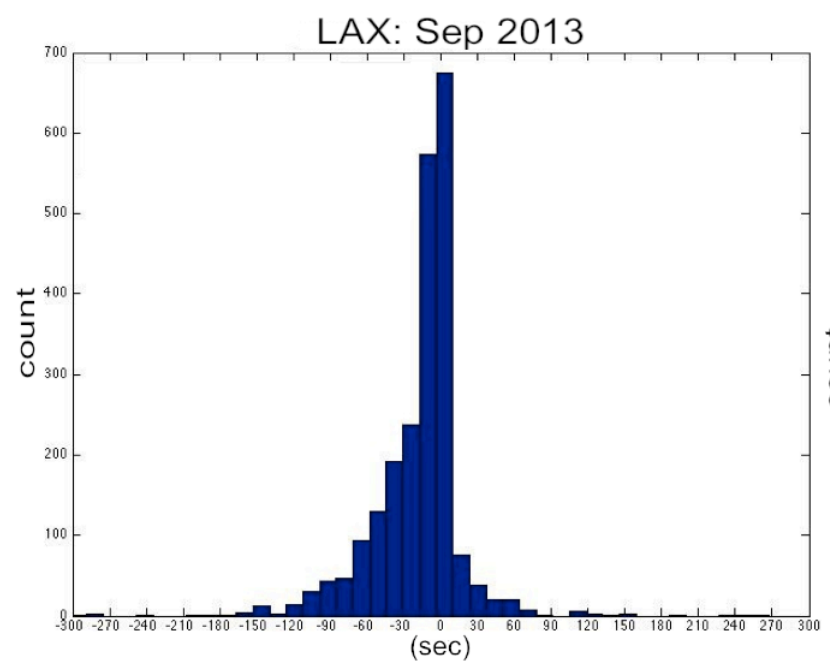

Figure 1. Undelayed Delivery Accuracy Histograms for all Four Airports across the Hottest Months. $a$.) $D E N$ in August 2013. b.) IAH in August of 2013 c.) LAX in September 2013 and d.) PHX in June of 2013

seconds with a standard deviation of 75.53 seconds. The figure and table illustrate that the majority of the aircraft were delivered to the meter fix under this methodology within \pm sixty seconds and that a large percentage of the aircraft were delivered within \pm thirty seconds.

The histograms in Fig. 1 show the number of aircraft and the manner in which they were distributed in delay across the airport meter fixes. Although DEN and IAH had a much larger percentage of aircraft remaining as a result of the filtering method, LAX had much larger set of aircraft landing, therefore the count values vary across the graphs. Therefore, DEN and LAX, due to the larger set of aircraft considered in the distribution, have a standard deviation slightly greater than IAH and PHX, accordingly, for the hottest month considered. The graphs for LAX and PHX shown in Fig. 1 illustrate a skewing of the delay distribution to the negative. This indicates that several of the nearly undelayed flights arrived earlier at the meter fix than scheduled in LAX and PHX. The delay values shown in Table 5 could be attributed to a number of factors including wind and the number of nearby airports, as well as airspace configuration. The positive mean values of undelayed delivery accuracy indicate the aircraft arrived slightly behind time due to the presence of a head wind and conversely the negative values could indicate flights arrived ahead of time possibly due to a tail wind. An inaccurate wind estimation in TBFM may account for some of the skewing of the data in the histograms, however additional investigation is required.

The data in Table 5 illustrate that the average nearly undelayed delivery accuracy ranged from 11.03 seconds to 27.78 seconds across all four airports and time periods considered. In addition, the standard deviation for the set data ranged from 40.61 seconds to 129.09 seconds.

The method of filtering data from the set of current operational data illustrated that this technique to compute delivery accuracy across a wide range of aircraft exists and can be completed in an automated fashion across airports in the NAS. Moreover, the data also provides additional information that allows the nearly undelayed delivery

8 
accuracy to be shown for each individual meter fix for a given airspace as well aircraft state data as the meter fix was crossed. This data can be leveraged for further investigation and development of a more refined automated filtering methodology. It should be noted, however, there were several facets of the data that prompt further exploration. Several of the airports showed skewing of delivery accuracy values to more negative values, as seen by several negative average undelayed delivery accuracy computations. The values could indicate biasing of the aircraft from the ARTCC into these airports or could be a result of the filtering itself.

\section{Conclusion}

The paper presented an automated approach to compute the nearly undelayed delivery accuracy of current day ATC operations. The approach has the advantage that it does not require a specific or already modified set of flight data, but can rather work with data from any site or time, based on the OTTR repository. This feature has the potential to aid in the definition of delivery accuracy metrics for future ground based scheduling tools and to help the transition of NextGen operations that rely on these metrics. The new method also enables the analysis of the undelayed delivery accuracy at meter points in order to understand changes of desired flow rates which allows aircraft to meet their STAs while performing high precision arrivals. The application of the filtering method to current data across four airports assures that the method can be utilized quickly and has the ability to function across various airspaces and adaptations.

The automated filtering also has the potential to be further refined in order to utilize the large amount of detailed information available in the data sets. A more detailed filtering method can be created and a more accurate delivery accuracy picture can be obtained across various ARTCCs using this additional information. In addition, the data can provide information about trajectories of aircraft that fall outside the bounds of the desired delay values as they cross the meter fix. This information can be used to develop methods that allow for delivery accuracy to be improved across a large set of aircraft. Using a large set of data, from IAH, DEN, LAX, and PHX from 2013. More data from different centers would be required to make sure that the test can be run for a wider range of operational environments. This could be a possible area for future work. More research is necessary to refine these metrics and to make sure that they correctly represent the delivery accuracy overall.

\section{Acknowledgments}

The authors thank Michelle Eshow from NASA Ames as well as Al Capps, MOSAIC ATM, and Jody Null at NTX for their support of the work and providing reference of previous tools in the area as well as facilitating access to the OTTR data set.

\section{References}

\footnotetext{
${ }^{1}$ Swenson, Harry, John E. Robinson III, Steve Winter, July 2013, NASA's ATM Technology Demonstration-1: Moving NextGen Arrival Concepts from the Laboratory to the Operational NAS, Journal of Air Traffic Control, pp. 22-44.

${ }^{2}$ Baxley, B., W. Johnson, H. Swenson, J. Robinson, T. Prevot, T. Callantine, J. Scardina, M. Greene, 2012, "Air Traffic Management Technology Demonstration-1 Concept of Operations (ATD-1 ConOps)," NASA/TM-2012-217585

${ }^{3}$ Swenson, H. N., Thipphavong, J., Sadovsky, A., Chen, L., Sullivan, C., and Martin, L., P., "Design and Evaluation of the Terminal Area Precision Scheduling and Spacing System," $9^{\text {th }}$ USA/Europe Air Traffic Management Research and Development Seminar, Berlin, Germany, June 2011.

${ }^{4}$ Thipphavong, J., Swenson, H. N., Lin, P., Seo, A. Y., and Bagasol, L. N., "Efficiency Benefits Using the Terminal Precision Scheduling and Spacing System," AIAA 2011-6971, $11^{\text {th }}$ AIAA Aviation Technology, Integration, and Operation (ATIO) Conference, Virginia Beach, Virginia, Sep. 2011.

${ }^{5}$ Prevot, H., B. Baxley, T. Callantine, W. Johnson, L. Quon, J. Robinson, H. Swenson, 2012, "NASA's ATM Technology Demonstration-1: Transitioning Fuel Efficient, High Throughput Arrival Operations from Simulation to Reality," HCI-Aero 2012, Brussels, Belgium

${ }^{6}$ L. Ren and J.P. Clarke, "Separation Analysis Methodology for Designing Area Navigation Arrival Procedures," Journal of Guidance, Control, and Dynamics, vol. 30, no. 5, pp. 1319-1330, 2007.

${ }^{7}$ NASA NTX, “Operational TMA/TBFM Repository (OTTR) Research Notebook," Sept. 2010.

8 , "Flight-test evaluation of the tool for analysis of separation and throughput," Journal of Aircraft, vol. 45, no. 1, pp. 323-332, 2008.

${ }^{9}$ S. Shresta and R. H. Mayer, "Benefits and constraints of time-based metering along RNAV STAR routes," in Proceedings of the Digital Avionics Systems Conference, 2009.

${ }^{10}$ FAA, "NextGen Implementation Plan," Jun. 2013.

${ }^{11}$ MITRE Center for Advanced Aviation System Development (CAASD), "Terminal Sequencing and Spacing (TSS) Concept of Operations," MP130330, Jul. 2013.
} 
${ }^{12}$ MITRE CAASD, “Terminal Sequencing and Spacing (TSS) Simulation 2 Plan and Procedures,” MP130132, Mar. 2013

${ }^{13}$ MITRE CAASD, “Terminal Sequencing and Spacing (TSS) Simulation 2 Post Analysis Report,” MP130272, Jun. 2013.

${ }^{14}$ Thipphavong, J., Jung, J., Swenson, H. N., Martin L., Lin M., and Nguyen, J., "Evaluation of the Terminal Sequencing and Spacing System for Performance-Based Navigation Arrivals," 32 ${ }^{\text {nd }}$ DASC, Syracuse, New York, Oct. 2013.

${ }^{15}$ International Civil Aviation Organization (ICAO), "Performance-based Navigation (PBN) Manual," $3^{\text {rd }}$ Edition, 2008.

${ }^{16}$ Erzberger, H., "Design Principles and Algorithms for Automated Air Traffic Management," AGARD Lecture series 200, AGARD-LS-200, Nov. 1995.

${ }^{17}$ Wong, G., "The Dynamic Planner: the Sequencer, Scheduler, and Runway Allocator for Air Traffic Control Automation," NASA TM-2000-209586, 2000.

${ }^{18}$ Prevot, T., "How to Compute a Slot Marker - Calculation of Controller-Managed Spacing Tools for Efficient Descent with Precision Scheduling," $31^{\text {st }}$ DASC, Williamsburg, Virginia, 2012.

${ }^{19}$ FAA, Terminal Procedure Publication, URL:

http://www.faa.gov/air_traffic/flight_info/aeronav/productcatalog/ifrcharts/TerminalProcedures/ 